\title{
The IGCS global curriculum mentorship and training program: building human capacity for gynecologic cancer treatment and research where the need is greatest
}

Thomas Randall (D) , Boston, USA; Linus Chuang, Danbury, USA; Soon Yau Joseph Ng, Singapore; Kathleen M Schmeler (D) , Houston, USA and Michael A Quinn, Melbourne, Australia

Women living in low- and middle-income countries lack access to timely diagnosis and treatment of gynecologic cancers. Deaths from ovarian and uterine cancers are comparable to those in high-income countries while $88 \%$ of deaths from cervical cancer occur in low- and middleincome countries. Doctors, nurses, and allied medical professionals are in short supply and opportunities for training in cancer care are limited. Those who train in high-income countries may find few opportunities in their home country or region, leading to medical 'brain drain', or 'human capital flight', in which highly educated and talented individuals from low-resource countries emigrate in search of personal or professional advancement. Training in high-income countries, furthermore, might not best prepare a provider to deliver cancer care in resource-limited settings.

The International Gynecologic Cancer Society (IGCS) recognized an opportunity to harness the talents and energies of its global membership to address this problem. Effective interventions require up-to-date curricula, stable relationships, and committed, sustained personal engagement. The IGCS Global Curriculum program pairs academic gynecologic oncologists in high-income countries with experienced clinicians in low- and middle-income countries to create structured gynecologic oncology fellowships. Starting with five sites in 2017, the program has now grown to 12 sites (Figure 1). These programs share core components, including twinning

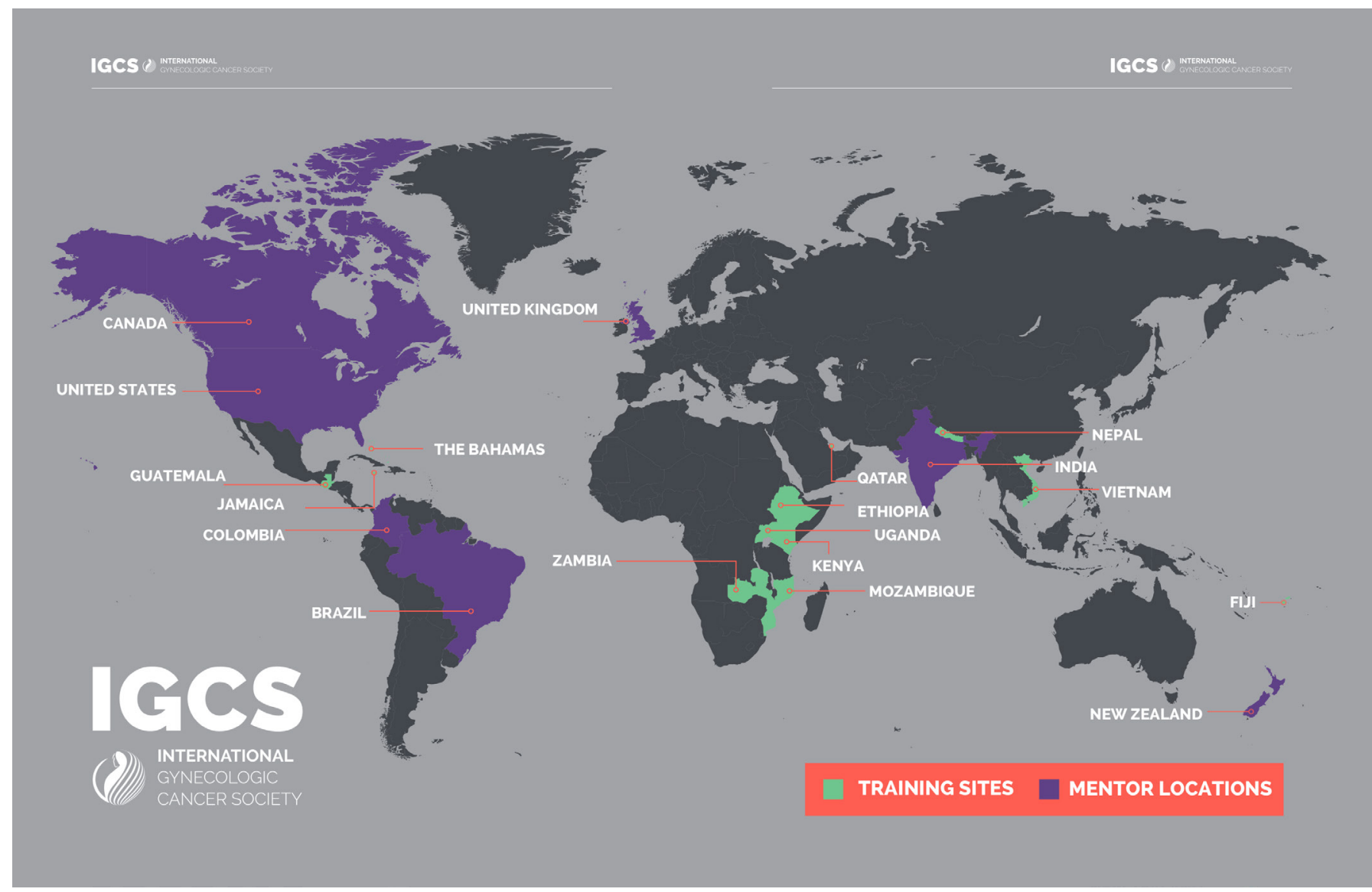

Figure 1 The location of the 12 fellowships in low- and middle-income countries as well as the countries of the international partnering institutions and mentors. 


\section{Corners of the world}

between the local site and an established program in a high-income country, virtual and hands-on training, structured evaluations, and examination for a certificate of completion. Most programs have arisen from established relationships between physicians and institutions in different parts of the world. To these existing programs, the IGCS, under the direction of its Global Curriculum and Mentorship committee, provides the curriculum, including regularly scheduled Project ECHO (Extension for Community Healthcare Outcomes) tumor boards, in which virtual videoconferencing is used for case review and didactic teaching. Fellows perform surgeries and provide clinical care with local and international mentors. The IGCS maintains an online REDCap database (www.redcap.org), in which the fellows can document their cases. Fellows receive formal, documented feedback from their mentors quarterly. When fellows document expected clinical and scholarly milestones they are invited to take an oral examination. To minimize the risk of bias due to variations in cultural norms the examination follows an Objective Standardized Clinical Examination format and is administered by examiners familiar with the setting and culture in which the fellow has been trained.

As of February 1, 2021, 41 fellows have performed more than 4473 procedures. Seven fellows graduated from the fellowship in 2019 and 2020. From the initial vision of training on site, the IGCS is now well on the way to building a generation of local champions to lead the fight against gynecologic cancers in low- and middleincome countries. More information can be found at https://igcs.org/mentorshipand-training/global-curriculum/.

Correspondence to $\mathrm{Dr}$ Thomas Randall, Cancer Center, Massachusetts General Hospital, Boston, MA 02114, USA; trandall@mgh.harvard.edu

Twitter Thomas Randall @trandallmd

Acknowledgements The authors would like to recognize Mary Eiken, CEO of the International Gynecologic Cancer Society, and Susan Ralph, for their tireless support of the program. We thank Mark Munsell for support of the program's REDCap database. Lastly, we thank the international and local mentors for their devotion to the fellows' training and to patient care.

Contributors All authors participated in the conception, planning, and editing of this manuscript equally. TR drafted the manuscript.

Funding The authors have not declared a specific grant for this research from any funding agency in the public, commercial or not-for-profit sectors.

Competing interests None declared.

Patient consent for publication Not required.

Provenance and peer review Commissioned; internally peer reviewed.

(C) IGCS and ESG0 2021. No commercial re-use. See rights and permissions. Published by BMJ.

\section{Check for updates}

To cite Randall T, Chuang L, Ng SYJ, et al. Int J Gynecol Cancer Published Online First: [please include Day Month Year]. doi:10.1136/ijgc-2021-002480

Accepted 25 February 2021

Int J Gynecol Cancer 2021;0:1-2.

doi:10.1136/ijgc-2021-002480

ORCID iDs

Thomas Randall http://orcid.org/0000-0002-32300310

Kathleen M Schmeler http://orcid.org/0000-00029670-4189 